\title{
Elicitation of US and Chinese expert judgments show consistent views on solar geoengineering
}

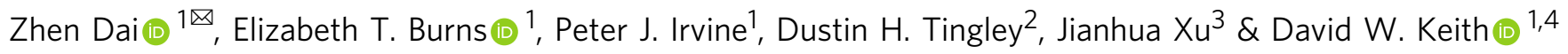

Expert judgments on solar geoengineering (SG) inform policy decisions and influence public opinions. We performed face-to-face interviews using formal expert elicitation methods with 13 US and 13 Chinese climate experts randomly selected from IPCC authors or supplemented by snowball sampling. We compare their judgments on climate change, SG research, governance, and deployment. In contrast to existing literature that often stress factors that might differentiate China from western democracies on SG, we found few significant differences between quantitative judgments of US and Chinese experts. US and Chinese experts differed on topics, such as desired climate scenario and the preferred venue for international regulation of SG, providing some insight into divergent judgments that might shape future negotiations about SG policy. We also gathered closed-form survey results from 19 experts with $>10$ publications on SG. Both expert groups supported greatly increased research, recommending SG research funding of $\sim 5 \%$ on average (10th-90th percentile range was $1-10 \%$ ) of climate science budgets compared to actual budgets of $<0.3 \%$ in 2018 . Climate experts chose far less SG deployment in future climate policies than did SG experts.

\footnotetext{
${ }^{1}$ John A. Paulson School of Engineering and Applied Sciences, Harvard University, Cambridge, MA, USA 02138. ${ }^{2}$ Government Department, Harvard University, Cambridge, MA, USA 02138. ${ }^{3}$ College of Environmental Sciences and Engineering, Peking University, Beijing, China 100871. ${ }^{4}$ John F. Kennedy School of Government, Harvard University, Cambridge, MA 02138, USA. ${ }^{凶}$ email: zdai@g.harvard.edu
} 


\section{Introduction}

olar geoengineering (SG), the large-scale deliberate manipulation of earth's radiative forcing (RF) to reduce climate hazards, has received increasing attention in climate science and policy. SG is politically contentious with active disputes about the scale and scope of research and deep uncertainties about the governance of deployment. Resolution of these disputes will depend, in part, on how SG is viewed by stakeholders with varying degrees of knowledge and diverse sets of interest (Wright et al., 2014). While at least 45 empirical studies have probed public opinion about SG, we found only six that probed the opinions of experts (Supplementary Table 1). Among these, most studied published documents (literature, policy documents, media coverage, etc.) to analyze themes and sentiments in SG, but only two (Winickoff et al., 2015; Dannenberg and Zitzelsberger, 2019) collected expert opinions directly, and none used structured elicitation procedures to assess the complex underpinnings of expert judgments. This imbalance is inappropriate. While informed public opinion should carry substantial weight in eventual decision-making about SG, surveying public opinion of low-visibility emerging technologies has its limitations. There is strong evidence that such views are far less stable than public views on topics with long-running high-visibility public debate (Zaller, 1992). Expert opinions are important because their judgments and issue framings influence media reporting which, in turn, influences more robust public opinions that will emerge if SG sees more public debate (Spruijt et al., 2014). In addition, experts typically have greater access to policy makers than do members of the public.

Studies of elicited expert judgments illuminate debates within expert communities. Expert elicitation has been used to support understanding of many areas of climate change with insufficient knowledge (Morgan, 2014). It is also an important method for evaluating uncertainties in Intergovernmental Panel on Climate Change (IPCC) reports (Oppenheimer et al., 2016), a key input to international climate negotiations. In this case, they may inform debate about governance of SG because experts have knowledge beyond that captured in journal literature and may play important roles in shaping international climate policies. Experts affect international policy coordination by sharing their knowledge with states (Haas, 1992) and may facilitate collaboration outside of official diplomatic channels (Stone, 2013) as when scientists from the US and Soviet Union formed networks to decrease nuclear tension (Evangelista, 2002).

SG is not a single technology, but rather a set of emerging scientific and technological capabilities whose climatic impacts cannot be confined within national borders. If used, deployment would entail design choices about objectives, methods, and amount of RF (Kravitz et al., 2016) which require international collaboration. The ability or inability of nations to collaborate on decisions about deployment will play a critical role in determining SG's benefits and harms (Reynolds, 2017). Cross-national comparison of expert opinions can provide early insight on both the degree of consensus and potential points of conflict.

We focus on experts from the US and China, the world's largest economies and highest carbon emitters that will play paramount roles in shaping international climate policy (Wuebbles et al., 2017). While voices from the breadth of the international community are needed, we choose US and China because of their weight in climate change issues. Furthermore, as two countries with widely different political system, culture, and socioeconomic realities, contrasting opinions between experts might also reveal issues emblematic of the sharpest cross-national conflicts regarding SG. Neither country has an official policy on SG, and expert opinions are opaque, particularly in China (Edney and Symons, 2014). Given the differences between China and the
US, it is particularly easy for researchers to focus on factors that might differentiate China from the US when exploring issues related to SG in China. Indeed, prior analyses of Chinese views about SG have highlighted factors that could lead deployment decisions to diverge from decisions made by western democracies. These include philosophical traditions (Moore et al., 2016; Wong, 2013) and histories of large engineering projects (Bluemling et al., 2019; Moore et al., 2016). However, it is difficult to gauge the importance of these factors in empirical decision-making processes if attitudes towards SG itself are unknown. We aim to reduce uncertainty by exploring expert judgments towards research, governance, and deployment of SG.

We focus on two aspects: (1) the views US and Chinese climate experts hold on SG and related topics, and (2) how different these views are. We conducted in-person semi-structured interviews with 13 US and 13 Chinese climate experts using a structured expert elicitation protocol (Morgan and Henrion, 1990) that combined quantitative and qualitative questions. This method limits the number of participants but provides an opportunity to understand detailed underpinnings of experts' judgments that larger-scale surveys such as, Dannenberg and Zitzelsberger (2019) typically do not allow. The structured format allows us to directly compare results between experts on a pre-defined set of questions in contrast to panel discussions such as Winickoff et al. (2015). Among our questions, some quantitative ones probed probabilistic judgments. The interviews cover three interrelated topics: (1) climate risks and emissions or RF trajectories, (2) the risks, benefits, and governance of SG deployment, and, (3) the scale, content, and governance of SG research. Experts were recruited by random draw from a list of each countries' authors for the IPCC Assessment Report (IPCC, 2014). The overall response rate was $19 \%$. Given the lower number of IPCC authors in China, we used snowball sampling starting from the IPCC author list to recruit participants. Information on Chinese participants is listed in Table 1 , and US participants are listed in Table 2 . We note that among experts we interviewed in China, university affiliated experts are over-represented and government research instituteaffiliated experts are under-represented compared to the overall IPCC authorship. However, the affiliation distributions are similar between experts from US and China among experts we interviewed. In addition, we report results from a closed-form survey with a subset of identical quantitative questions administered to SG experts. This survey includes 19 out of the 28 SG experts who have 10 or more publications on SG. While we note that the online survey format is notably different from the faceto-face interview format, the overlapping questions from both are identical with detailed wording, and we expect the results to allow some comparisons between climate experts and experts most engaged in SG research.

Climate change: risks and emissions trajectories. Figure 1 summarizes answers to quantitative questions. Supplementary Fig. 2 includes analysis of the internal consistency of experts' responses. Plots with more detailed statistics are shown in boxplots in Supplementary Figs. 3-10. The interview guide is included in the supplementary, and we refer to questions by number when discussing results (e.g. Q1 refers to Question 1).

We first asked experts to rank the relative importance of climate hazards for their country (Q1). These questions also served to prime experts to think about specific hazards when answering later questions about SG (Dawson et al., 2015). Mean rankings were highest for increasing frequency and intensity of extreme heat and many justified their ranking by pointing to the ubiquity and degree of the impact ("top-ranked items are 


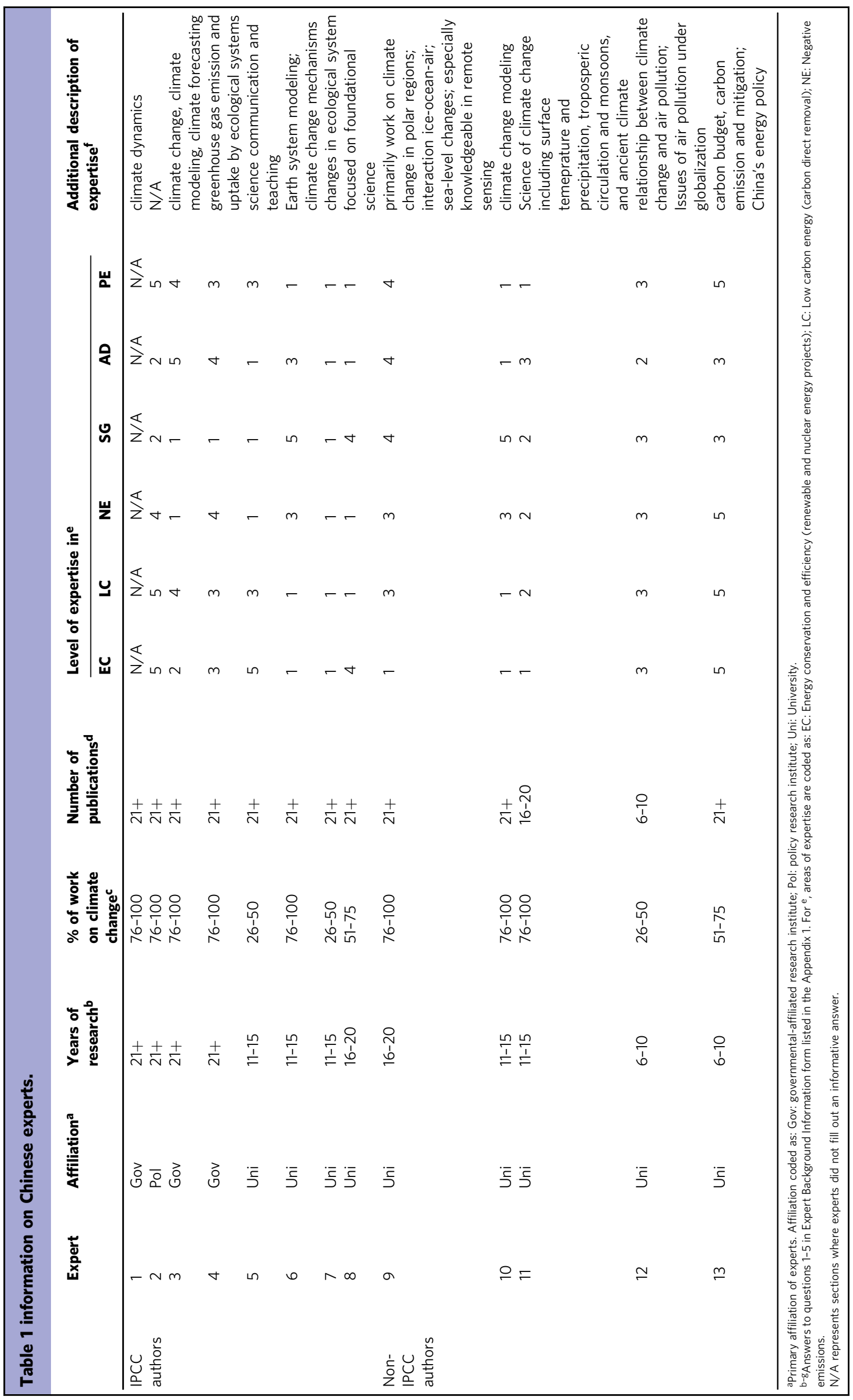


Table 2 Names and affiliations of US experts.

\section{Expert}

Alan Robock*

Richard Norgaard

Haroon Kheshgi

Patrick Kinney

Jonathan Wiener

Christopher Sabine

Karen Fisher-Vanden

Chris Forest

Phil Duffy

Virginia Burkett

Geoff Heal

Robert Lempert*

David Victor ${ }^{\star}$

Experts who have authored peer reviewed articles on SG are marked with "*t.

impactful where people live"; "items are ranked based on environmental, economic, and political impacts"). Ranking was lowest for sea level/ sea ice and ocean acidification with experts stating that these factors might be important globally, but not to the US or China this century. Experts were generally uncertain about their rankings, pointing out that the hazards overlap and interact. The only statistically significant difference between US and Chinese rankings were for extreme precipitation and inland flooding, ranked most important by Chinese and least important by US experts. Chinese experts referred to the lack of inland infrastructure to properly divert away floods as well as large impact on economic activity and loss of human lives for the high importance. In contrast, US experts pointed to the higher population density and property value in coastal regions as reasons to downrank this option and ranked sea level rise, coastal flooding including storm surges higher than did Chinese experts.

We then asked experts questions about China's emissions trajectories (Q2). Chinese experts estimated lower emission values for China's 2075 carbon emissions, lower peak emissions, and an earlier Chinese emissions peak, than did US experts. However, differences were small, with statistically significantly differences only for the upper bound estimates. Chinese experts expressed trust in the government's stated goals in the Nationally Determined Contribution to the Paris Agreement (emissions peaking by 2030) while US experts emphasized economic and population growths as factors that might delay the emissions peak.

Estimates for 2075 RF (Q3) are consistent between US and Chinese experts. The mean estimate of $4.3 \mathrm{~W} / \mathrm{m}^{2}$ is close to the mean of the representative climate pathways (RCP) 4.5 scenario. Since the RCP scenarios were presented during the interview, many experts mentioned that they based their answers on the RCP scenarios, ("I think we are doing better than RCP 4.5 and the worst-case scenario would not exceed RCP 8.5 "). One important difference is that US experts had a broader range of estimates than Chinese experts: their 10th-90th percentile estimates were both inside of the US experts' range.

Deployment: strategy, risks, governance, and nationality. We also asked experts to specify their desired $2075 \mathrm{RF}$ and how much SG RF they would use to achieve their RF goals (Q10) assuming they were tasked with designing a climate policy while considering realistic tradeoffs between costs of emissions reduction (including carbon dioxide removal (CDR)) and climate impacts. In sharp contrast to the broadly consistent estimates about emissions trajectories and climate risks, US experts' desired RF is statistically significantly lower than Chinese experts' by $1.3 \mathrm{~W} / \mathrm{m}^{2}$.
As a result, the difference between the expected and the desired $\mathrm{RF}$ is statistically significantly higher in the US case, signifying a more aggressive climate goal. However, few in either group choose to use SG to achieve their goals: only two US and two Chinese experts chose to use SG to offset RF by 2075. However, two additional US experts said that they would consider using SG in some capacity before 2075 , but thought SG RF should decrease to 0 by 2075 .

US and Chinese experts generally agreed on the most important risks of SG (Q15). On average, more than half of experts think that impact on regional climate (including monsoon) is the most important uncertainty in physical impact. For the most important physical hazard, stratospheric ozone impact was chosen by Chinese experts, while other environmental impact of implementation was chosen by US experts. Experts were told to include "unknown unknowns" in this category. Experts who chose ozone impact suggested they were primarily thinking about stratospheric aerosol injection. US and Chinese experts differed about the most important socio-economic risks, with US experts choosing difficulties of governance as the most important while Chinese chose conflicts caused by varied regional outcomes.

US and Chinese experts both prefer international governance over governance by a consortium of states or no international governance (Q11). Chinese and US experts differed about the venue for governance with all Chinese experts preferring extension to the United Nations Framework Convention on Climate Change (UNFCCC), while US experts prefer governance by a new formal UN treaty structure. US experts point to the slow decision process and difficulties in achieving consensus as reasons to move away from the UNFCCC ("UNFCCC is so fraught with bureaucracy at the moment that it will be incapable of properly governing", "UNFCCC has important processes for norm-setting but it's hard to do anything aggressive with consensus of $100+$ countries") while Chinese experts stress that the existing UNFCCC processes are the easiest to follow ("a new framework won't be very different from UNFCCC and is more costly to set up"). One expert from each country did not choose from the provided options. One of these experts suggested that it was premature to choose a governance option, and that international scientific collaborations similar to CERN should be promoted first. Another suggested "norm-driven responsive use or tacit governance", explaining it as "cooperation by observing each other's behavior and adjusting our behavior accordingly".

Experts had little confidence in assessing the probability that the US or China would be among the first nations to deploy SG even if SG were shown to be effective and technical issues related to deployment were resolved (Q12). Experts from both countries think it is a coin flip ( $\sim 50 \%$ probability on average) and the spread of answers is large (standard deviation for the probability is $34 \%$ ). Experts arguing for the possibility of deployment suggested reasons including desire to be included in an international SG effort, technological capability, and climate emergency framing. Experts arguing against it suggested reasons including uncertainties in outcome and risk aversion ("the worst thing to happen if SG is implemented is global nuclear war...the US wouldn't do it unless there was a global agreement"; "China will be very cautious if risks and uncertainties remain"). However, many experts said that they simply did not know.

No expert expected cultural factors to have significant impact on the probability of SG deployment by their country $(\mathrm{Q} 17,18)$. However, experts defined the term "culture" in different ways and offered a range of potentially important factors as shown in Supplementary Table 2. The cultural factors identified by the two groups of experts had little overlap. Most prominently, US experts gave a less diverse range of answers and mostly pointed to the difference in political processes, while only one Chinese expert 


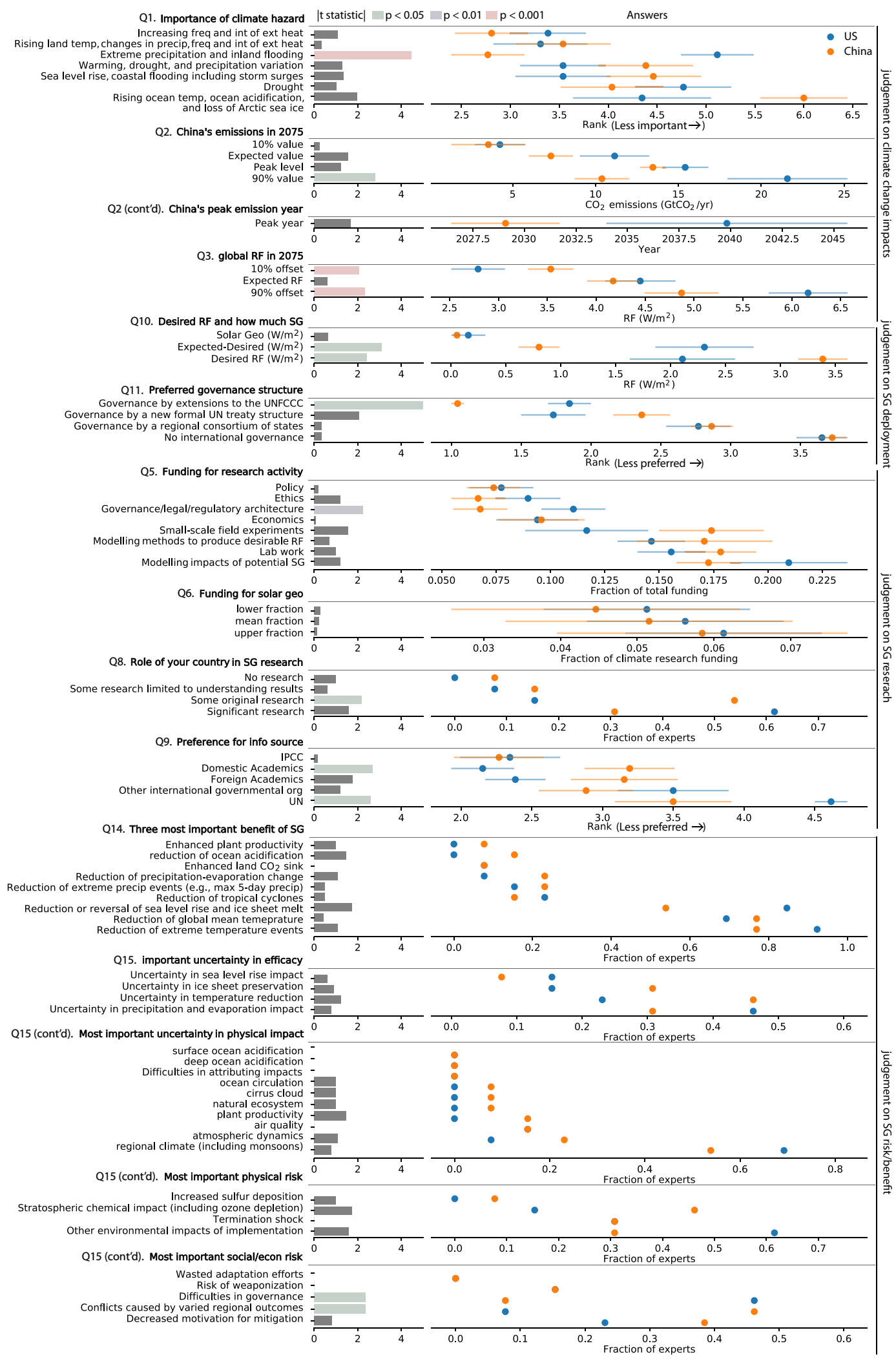

Fig. 1 Answers to quantitative questions and variations between US and Chinese experts. (Right) Scatter plots of quantitative answers and (left) bar plots of absolute values of $t$ statistics showing differences between US and Chinese experts' answers. Scatter plots show the average answers of US and Chinese experts. The error bars represent standard deviations of the population mean estimated from experts' answers. Answers with statistically significant differences between US and Chinese experts are highlighted on the bar plots at different significance levels. Answers within each question are arranged by descending values of the mean of pooled answers. 
Table 3 Summary of discussions on SG research.

\begin{tabular}{|c|c|c|}
\hline Research areas & US experts & Chinese experts \\
\hline Deployment technology & $\begin{array}{l}1 \text { expert supported research into deployment technology; } \\
2 \text { experts explicitly said no research into deployment } \\
\text { technologies }\end{array}$ & 3 experts supported research into deployment technology \\
\hline $\begin{array}{l}\text { Large-scale outdoor } \\
\text { research }\end{array}$ & $\begin{array}{l}\text { No expert thinks large scale experiment should be done in the } \\
\text { short term; } \\
4 \text { mentioned the possibility of longer-term larger scale } \\
\text { research but only if other smaller scale research can't answer } \\
\text { the questions and if major governance is put in place }\end{array}$ & 1 expert support large scale engineering demo \\
\hline $\begin{array}{l}\text { Small-scale outdoor } \\
\text { research }\end{array}$ & $\begin{array}{l}7 \text { experts mentioned small scale research with caveats, (e.g. } \\
\text { needs independent governance) }\end{array}$ & $\begin{array}{l}3 \text { experts mentioned small scale research with caveats } \\
\text { (e.g. needs independent governance) }\end{array}$ \\
\hline A range of methods & $\begin{array}{l}7 \text { experts explicitly mentioned research into a range of } \\
\text { technologies (e.g., marine cloud brightening, space-based SG } \\
\text { technologies) }\end{array}$ & $\begin{array}{l}3 \text { experts mentioned research into a range of technologies } \\
\text { (e.g., marine cloud brightening, space-based SG } \\
\text { technologies) }\end{array}$ \\
\hline
\end{tabular}

mentioned this aspect. Three experts in each country said that culture would have no impact on SG deployment.

Experts also responded differently regarding the impact of past engineering projects on SG deployment. They were first asked to list projects that reminded them of SG in an open-ended question (Q19). Seven Chinese experts referred to domestic engineering projects, while US experts exclusively mentioned international projects. We then gave them a list of past domestic engineering projects and asked if any would affect SG deployment (Q20). All but one Chinese expert selected at least one domestic engineering projects, while only five US experts did.

Research: funding, structure, and regulation. Experts were asked qualitatively what SG research they did like to see and were given the option to support "no research" (Q4) before we presented them with more details on research activities and asked them to make quantitative judgments on funding $(\mathrm{Q} 5,6)$. A summary of qualitative research ideas is shown in Table 3. Quantitatively, experts assigned an average of 5\% of climate research funding to a loosely coordinated SG research program by 2030. The median value is $4 \%$ (Q6). When asked about the funding assignment for specific research activities (Q5), experts in the US and China both assigned more funding to natural science research activities (66\% on average) compared to social science research activities. The fraction of funding assigned ranges from $7 \%$ for ethics research to $21 \%$ for modeling impacts of potential SG activities. In addition to the funding, we also asked experts to assign importance to research activities, recognizing that some research activities would cost more, even if they were similarly important. This effect was not large: on average, natural science research was assigned $62 \%$ of importance.

In terms of roles that US and China should play in SG research (Q8), only one expert suggested that no SG research should be done. Most US experts selected significant research leading the development of the field for the US's role in SG research while most Chinese experts selected some original research contributing to the development of the field for China's role in SG research.

The IPCC was the most preferred information source for SG on average, but US experts prefer domestic academics to the IPCC. The UN was the least preferred information source for both US and Chinese experts. US experts trusted domestic academics significantly more than the UN (Q9).

Just over half of the experts (9 US and 6 Chinese) said no regulation is needed for SG research if standard health and safety rules were already considered (Q7). Others mentioned that additional regulations were needed as summarized in Supplementary Table 3. Experts from both countries mentioned that there should be some rules that require experiments to be contained (e.g. within the laboratory) wherever possible, although some added that there may be some cases when outdoor experiments are needed to resolve questions that cannot be solved indoors.

\section{Discussion}

Perhaps the single most striking result was the broad agreement between US and Chinese experts. Results in Figs. 1 and 2 show strong overall agreement between US and Chinese experts. We explored the distinction between experts systematically using hierarchical agglomerative clustering analyses applied across all quantitative questions (see "Methods" section). While we find clustering among sub-questions (as expected) we find no clustering of experts by nationality (Supplementary Fig. 1). This empirical result is at odds with most of the prior literature that has speculated on the future diversion between US and Chinese SG policy by highlighting first factors that differentiate China from other countries. For example, Moore et al. (2016) suggested that China is unlikely to be the first to deploy SG because of its philosophical tradition. Wong (2013) explored Confucianism text related to public engagement and emphasized the lack of liberal democratic values in Chinese culture. Experts have strikingly consistent judgments on the trends of climate change, funding, and makeup of SG research program, and potential deployment scenarios.

Our results also contribute to a small but growing literature of empirical research on SG in developing countries. Findings of Chinese experts' willingness to increase SG research funding accord with a growing literature on public and expert opinions on SG that shows that experts and lay people are open to the idea of SG (Visschers et al., 2017; Dannenberg and Zitzelsberger, 2019; Sugiyama et al., 2020; Winickoff et al., 2015; Carr and Yung, 2018), albeit with caution.

If judgments from experts we interviewed reflect or predict emerging judgments among broader climate expert communities in each country, there would be reasonable common ground for collaboration and potential negotiation between the US and China on SG for both research and potential deployment. In addition, for countries with divergent political systems and histories such as the US and China, these commonalities could facilitate conversations among experts outside official political processes to enhance collaborations and promote diplomatic relationships (Stone, 2013). 

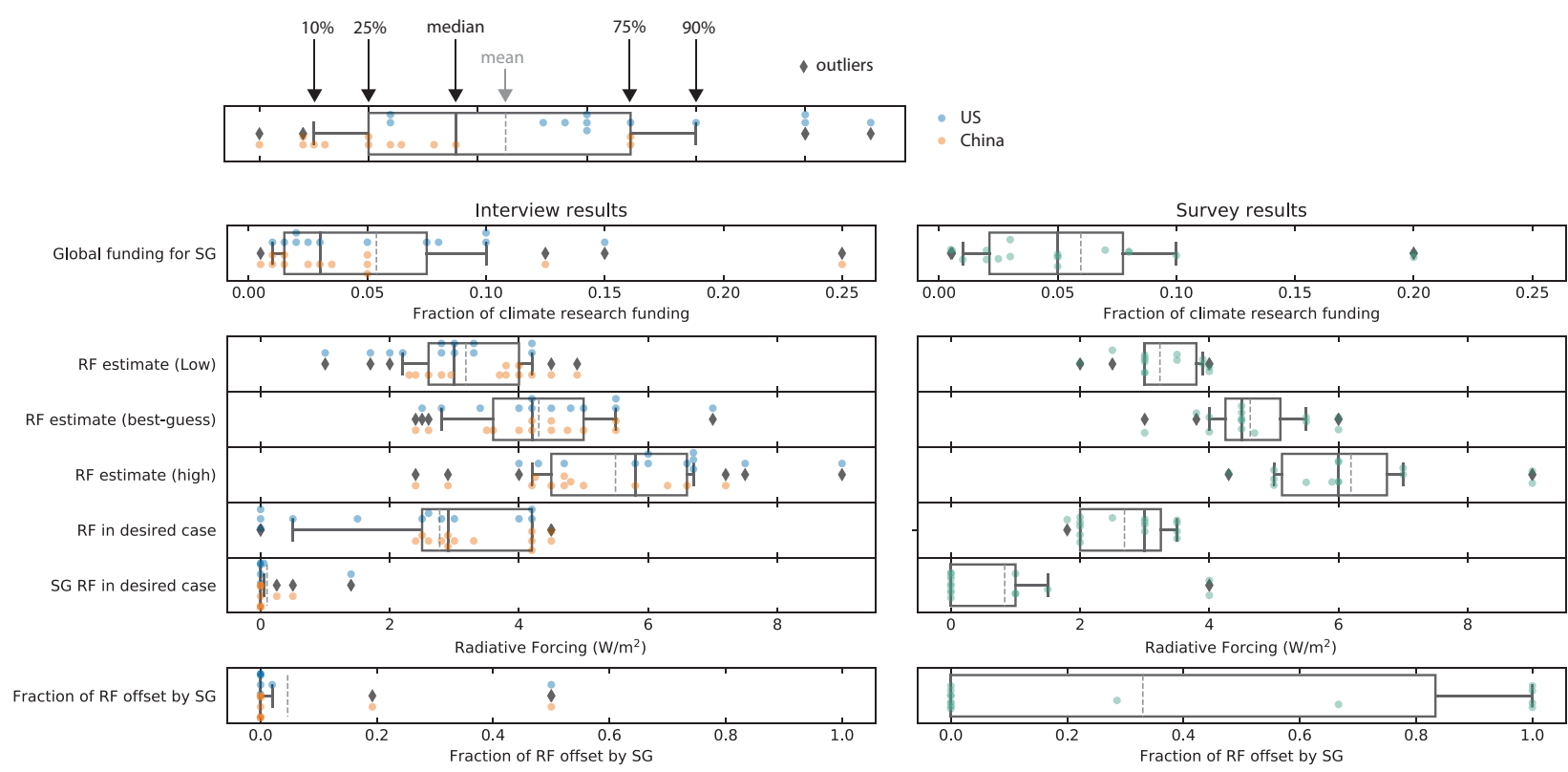

Fig. 2 Expert judgment on SG research program funding and radiative forcing. The results are shown in comparison to ones obtained by survey of SG experts who presented the same questions to a group of $18 \mathrm{SG}$ experts. (Top) A schematic of the Box-Whisker plot shown in this work. Plots are made with pooled answers from both US and Chinese experts. Outliers are identified as data outside of the 10th and 90th percentile values. Answers from US (blue) and Chinese (orange) experts are plotted as individual data points. The vertical displacements of these data points are introduced only to avoid overlap. (Bottom) Distribution of participant answers to question 6: "How much should be spent on the loosely coordinated solar geoengineering research program by 2030 as a percentage of current climate funding?" as well as questions 3 and 10 addressing participants' estimated, desired, and SG radiative forcing in 2075. The fraction of RF offset by SG is calculated based on participants' desired SG RF and their best-guess expected RF estimate in 2075 as SG RF/ (Expected RF-Desired RF). Results from a related survey of SG experts are shown in the right column for comparison. The fraction represents the amount of SG participants are willing to deploy to achieve their RF goals when their desired RF is lower than their expected RF.

The climate experts we interviewed from both countries unanimously support higher levels of funding for SG. This is consistent with the survey outcomes from SG experts. The amount of funding experts assigned to $S G$ research as a fraction of global climate research funding has a mean value of $5 \%$ and a median value of $4 \%$. Only one Chinese expert initially indicated that they preferred no research, but later specified that this applied to their own research effort, not the entire climate research community. The expert also clarified that they would not block funding for others and assigned $1 \%$ of total climate research funding to SG research. In comparison, global funding for SG research was roughly $\$ 8$ million in 2018 (Necheles et al., 2018), which accounted for $<0.3 \%$ of climate science funding in the US (US Government Accountability Office, 2018). Figure 2 shows the comparison between the interview result and the survey of self-identified SG experts. The mean and median results from the survey outcomes are both $2 \%$ higher than the interview outcomes, but the differences are not statistically significant. These similarities also give us some confidence that the survey results can be compared to the interview results despite the difference in question format. A recent study found that roughly half of climate experts in IPCC and UNFCCC support more investment in R\&D of SG technologies (Dannenberg and Zitzelsberger, 2019) in contrast to our finding of unanimous agreement on increased SG research funding. Several factors might explain this difference. First, experts in Dannenberg et al were not informed of the current level of funding for SG research. Second, Dannenberg et al. asked about degree of agreement with increased funding, whereas in our study allowed Experts who prefer low funding levels to indicate a specific non-zero number. Third, we asked for funding levels for SG research in general specifying that did not include developing deployment hardware, Dannenberg et al. asked participants about " $R \& D$ for geoengineering technologies" and it's plausible that their experts interpreted this as deployment technologies. We explicitly separated deployment technology from other research and found much lower support for developing deployment hardware (Table 3), which may explain part of the discrepancy.

Despite broad support for research, experts in both countries showed little support for SG deployment. Only four experts saw any prospect for SG deployment by 2075 . This is consistent with prior unpublished expert elicitation of IPCC authors showing that there was broad agreement that SG might reduce risk but experts were reluctant to consider its deployment (Carr et al., 2013). Desired SG RF (amount of RF reduction achieved through SG in experts' desired RF scenario) in 2075 has a slight correlation with China's expected $\mathrm{CO}_{2}$ emissions level in 2075 and expected $\mathrm{RF}$ in 2075 (Supplementary Fig. 2), indicating that there could be a higher level of SG deployment if there is insufficient mitigation action or if climate impact worsens. However, SG RF does not correlate with the difference between desired and expected RF in 2075, showing that the difficulty of achieving expert's desired level of future RF is not a key factor in experts' consideration. This result contrasts starkly with findings from the survey as shown in Fig. 2. Of the 19 SG survey participants, six indicated non-zero desired SG RF in 2075. This is consistent with the view that the SG expert community is biased towards deployment compared to their peers in other fields of climate science. Despite the general agreement on most issues, experts showed some disagreement on the aggressiveness of climate goals and preferred governance structure. US experts showed a more aggressive stance towards climate action and trust the current UNFCCC processes less in coordinating SG research activities. These might create contention in future negotiation. Differences also exist between the US and China caused by cultural factors and past experiences with engineering projects, but experts thought they would only play a minor role in SG deployment. 
Our work hints at a basis for US-China collaboration on SG as climate experts agree on a broad range of issues despite some striking differences. In particular, there is unanimous agreement that funding for SG research should increase, even though experts remain very cautious about deployment. These results are surprising given that prior literature assumed sharply different levels of support for SG research and focused on factors that might cause Chinese and US decisions on SG to diverge.

\section{Methods}

Expert selection process. Prior to the expert recruitment, the interview materials and methodologies were reviewed and approved by the Institutional Review Board (IRB) at participating universities.

For Chinese experts, we sent interview invitation emails to 43 experts whose affiliation was listed as "China" in IPCC AR5 report author list (IPCC, 2014). Because of the relatively small number of total IPCC authors in China, we did not expect to obtain consent from more than 10 authors. Therefore, in these emails, we asked experts to recommend additional experts who might be eligible to participate in the interview project. We then sent invitations to these recommended experts. We also sent at least one round of follow-up emails to experts who did not reply initially. We were able to interview eight experts who are IPCC authors and five experts who are not IPCC authors. Information about these experts is listed in Table 1 . We do not include names and institutions for Chinese experts to protect these experts' privacy and ensure their willingness to give truthful answers without worry of repercussions. This is also a common practice among elicitation projects in China.

The recruitment process for the US experts are the same as in the Chinese case with one difference: we sent invitations to a subset of 70 randomly selected US IPCC AR5 authors without accepting recommendations for non-IPCC authors. This is because there are 182 US IPCC authors-a much larger number than in the China case, and the acceptance rate is high enough that we did not have to reach out to experts outside of the IPCC author list. We initially sent out invitations to 50 randomly selected IPCC authors, followed by batches of 10 invitations until we obtained consent to interview 13 IPCC authors.

One notable bias in our sample is the over-representation of experts affiliated with universities and underrepresentation of those from government-affiliated institutions in China compared with the IPCC authorship. Among the overall Chinese IPCC authors, 88\%, $28 \%$, and $7 \%$ are affiliated with government-affiliated research institutions, universities, and other policy institutes, respectively. Among those we interviewed, the corresponding numbers are 3 $(23 \%), 9(69 \%)$, and $1(8 \%)$. For the 60 US experts that we sent invitations to, 19 (32\%), $37(62 \%)$, and $4(7 \%)$ are affiliated with government-affiliated research institutions, universities, and other policy institutes, respectively. Among those we interviewed, the corresponding numbers are 1 (8\%), 9 (69\%), and $3(23 \%)$.

Interview format. Interviews were conducted in sessions that ranged between 1 and $2.5 \mathrm{~h}$ based on prepared interview guides (see Supplementary). Interviews in China were conducted by Zhen Dai and Jianhua $\mathrm{Xu}$ in Chinese between June and August 2018, and interviews in the US were conducted by Zhen Dai and Elizabeth Burns in English between October 2018 and May 2019. The interview guides were in Chinese and English, respectively, and were translations of each other. There are some minor differences in question design between the US and Chinese interview guides, and only results from identical questions are included in this manuscript. Specifically, US experts were asked of the world's projected carbon emissions in Q2 while the Chinese experts were not.
In addition, Q13 is different between the two interviews. These changes were made with the US version because we wanted to obtain additional data on global emissions and improve the way Q13 was asked in the US version. During the interviews, each interviewee was given a copy of the interview guide so they could reference the exact language of the questions asked if needed. The interviewers took extensive notes during the interviews, and the interview recordings were transcribed for further analysis.

Data analysis. To compare the answers between US and Chinese experts, we conduct hypothesis testing where the null hypothesis is that US and Chinese experts have the same opinions. Student $t$ tests were conducted for all questions with quantitative answers. One other statistical test was also conducted for each question to validate the results. Mann-Whitney rank-order tests were conducted for questions that required numerical answers (Questions $2,3,5,6,10$ ) or rankings (Question $1,9,11$ ), and $z$-tests were conducted for questions that required selections of one or more items from given options (Question 8, 14, 15, 15). For answers to ranking questions, equal rankings were rescaled to preserve the rank-sum (e.g if a participant was asked to rank four items, a ranking of 1, 2, 2, 3 would be rescaled as $1,2.5,2.5,4$ so that the rankings from any one participant would sum to 10). For selection questions, answers were converted to one-hot encodings. We set the significance threshold for a two-tailed $p$ value of 0.05 .

Simpson's correlation coefficients were also calculated for questions with quantitative answers using the same data encoding. For hierarchical agglomerative clustering (HAC) (Rokach and Maimon, n.d.) analysis shown in SI Fig. 1, answers for each question were standardized to a mean of 0 and a standard deviation of 1. Two HAC analyses were done, one clustering experts, and the other clustering questions. For the former, answers to various questions from a specific expert was treated as a vector for clustering. For the latter, all experts' answers to a specific question was treated as a vector for clustering. Group-averaged Euclidean distances were used to calculate cluster similarities.

Occasionally, experts did not provide an answer to a question either because they found the question too hard to answer or because none of the options provided by us seemed viable. These answers were treated as missing values and omitted from the statistical tests and when calculating Simpson's correlation coefficients. For HAC, missing values were imputed as the sample mean.

Appropriate corrections to statistical significance levels are typically needed for multiple comparisons. We did not apply these corrections for hypothesis testing because our central finding was that the two groups of experts agree (the null hypothesis), and we want to highlight potentially divergent judgments and minimize Type II error. Neither did we apply corrections in the correlation analysis, because we want to scrutinize potentially inconsistent judgments.

Survey methodologies. In addition to semi-structured interviews, we elicit expert judgments about SG research priorities from a group of natural science researchers working on this topic using a structured formal survey and compare answers from that group to the interview results. The survey includes quantitative questions as well as free-form text responses including a request that participants provide specific research proposals. Some questions used in the survey are identical to Question 3, 6, and 10 in the interview, and answers to these questions are included in this study.

The survey participants were recruited from attendees of the 2018 American Geophysical Union (AGU) Fall Meeting. All authors of AGU abstracts that mentioned SG, whose contact details could be found, were contacted to see if they would be interested in participating. In addition, we sent emails to several of their more senior personal contacts in the field of SG as well as 
to other researchers working on climate science whose expertise would be applicable to SG. All who were interested were invited to complete the survey.

The survey received 61 responses. Supplementary Table 4 lists all 61 participants. We subsampled within this group to select participants who have high expertise in SG. Nineteen participants have published 10 or more peer-reviewed journal articles on the subject as determined by an analysis of the records which satisfied a Web of Science search on the 20 May 2020 and after merging duplicate authors. The specific search term was: TOPIC: ("solar climate engineering" or "solar geoengineering" or "solar radiation modification" or "solar radiation management" or "stratospheric aerosol geoengineering" or "marine cloud brightening" or "marine sky brightening" or "cirrus cloud thinning" or "geomip"). These participants represent 19 out of 28 total experts who have published 10 or more journal articles on SG according to this search. Only answers from these participants are included in the main figures. Effects of setting different numbers of publications as the subsampling threshold is shown in Supplementary Fig. 12. Changing the citation count threshold for sampling did not change the averages of any answer significantly but had a large impact on the median answers of the desired SG RF in 2075. We therefore refrain from drawing conclusions from the median statistic of the survey outcome for this question.

\section{Data availability}

The datasets generated during the current study (interview transcripts) are not publicly available due to confidentiality agreement with interviewees but are available from the corresponding author on reasonable request.

Received: 21 July 2020; Accepted: 7 December 2020;

Published online: 19 January 2021

\section{References}

Bluemling B, Kim RE, Biermann F (2020) Seeding the clouds to reach the sky: will china's weather modification practices support the legitimization of climate engineering. Ambio 49(1):365-73. https://doi.org/10.1007/s13280-01901180-3

Carr WA, Preston CJ, Yung L, Szerszynski B, Keith DW, Mercer AM (2013) Public engagement on solar radiation management and why it needs to happen now. Clim Change 121(3):567-577. https://doi.org/10.1007/s10584-013-0763-y

Carr WA, Yung L (2018) Perceptions of climate engineering in the South Pacific, Sub-Saharan Africa, and North American Arctic. Clim Change 147 (1-2):119-132. https://doi.org/10.1007/s10584-018-2138-x

Dannenberg A, Zitzelsberger S (2019) Climate experts' views on geoengineering depend on their beliefs about climate change impacts. Nat Climate Change 9 (10):769-775. https://doi.org/10.1038/s41558-019-0564-z

Dawson E, Hartwig M, Brimbal L (2015) Interviewing to elicit information: using priming to promote disclosure. Law Hum Behav 39(5):443-450. https://doi. org/10.1037/lhb0000136

Edney K, Symons J (2014) China and the blunt temptations of geo-engineering: the role of solar radiation management in China's strategic response to climate change. Pac Rev 27(3):307-332. https://doi.org/10.1080/09512748.2013.807865

Evangelista M (2002) Unarmed forces: the transnational movement to end the cold war. Cornell University Press, Ithaca, London

Haas PM (1992) Introduction: epistemic communities and international policy coordination. Int Organ 46(1):1-35. https://doi.org/10.1017/S0020818300001442

IPCC (2014) Fifth Assessment Report (AR5) authors and review editors. IPCC. https://www.ipcc.ch/site/assets/uploads/2018/03/inf07_p32_ipcc_ar5_ authors_review_editors.pdf

Kravitz B, Macmartin DG, Wang H, Rasch PJ (2016) Geoengineering as a design problem. Earth Syst Dyn 7(2):469-497. https://doi.org/10.5194/esd-7-469-2016

Moore JC, Chen Y, Cui X, Yuan W, Dong W, Gao Y, Shi P (2016) Earth's future special section: will China be the first to initiate climate engineering? Earth's Futur. https://doi.org/10.1002/eft2.169

Morgan MG (2014) Use (and abuse) of expert elicitation in support of decision making for public policy. Proc Natl Acad Sci USA 111(20):7176-7184. https://doi.org/10.1073/pnas.1319946111
Morgan MG, Henrion M (1990) Uncertainty: a guide to dealing with uncertainty in quantitative risk and policy analysis. Cambridge University Press, Cambridge

Necheles E, Burns E, Chang A, Keith D (2018) Funding for solar geoengineering from 2008 to 2018. Sol Geoeng Res Blog. https://geoengineering.environment. harvard.edu/blog/funding-solar-geoengineering

Oppenheimer M, Little CML, Cooke RM (2016) Expert judgement and uncertainty quantification for climate change. Nat Clim Change 6(5):445-451. https://doi. org/10.1038/nclimate2959

Reynolds JL (2017) Solar climate engineering, law, and regulation. In: Brownsword R, Scotford E, Yeung K, (eds) Vol. 1. Oxford University Press. https://doi.org/ 10.1093/oxfordhb/9780199680832.013.71

Rokach L, Maimon O (n.d.) Clustering methods. In: Data mining and knowledge discovery handbook. In: Maimon O, Rokach L, (eds). New York, Springer-Verlag, pp. 321-352. https://doi.org/10.1007/0-387-25465-X_15

Spruijt P, Knol AB, Vasileiadou E, Devilee J, Lebret E, Petersen AC (2014) Roles of scientists as policy advisers on complex issues: a literature review. Environ Sci Policy 40:16-25. https://doi.org/10.1016/j.envsci.2014.03.002

Stone D (2013). Knowledge actors and transnational governance. Palgrave Macmillan UK, London. https://doi.org/10.1057/9781137022912

Sugiyama M, Asayama S, Kosugi T (2020). The north-south divide on public perceptions of stratospheric aerosol geoengineering?: a survey in six AsiaPacific countries. Environ Commun 1-16. https://doi.org/10.1080/1752403 2.2019.1699137

US Government Accountability Office (2018). Analysis of reported federal funding. US Government Accountability Office

Visschers VHM, Shi J, Siegrist M, J Arvai J (2017). Beliefs and values explain international differences in perception of solar radiation management: insights from a cross-country survey. Clim Change https://doi.org/10.1007/ s10584-017-1970-8

Winickoff DE, Flegal JA, Asrat A (2015) Engaging the global south on climate engineering research. Nat Clim Change 5(7):627-634. https://doi.org/ $10.1038 /$ nclimate 2632

Wong P-h (2013) The public and geoengineering decision-making. Techné 17 (3):350-367. https://doi.org/10.5840/techne201421110

Wright MJ, Teagle DAH, Feetham PM (2014) A quantitative evaluation of the public response to climate engineering. Nat Clim Change 4(2):106-110. https://doi.org/10.1038/nclimate2087

Wuebbles DJ, Fahey DW, Hibbard KA, Dokken DJ, Stewart BC, TK Maycock TK (2017). Climate science special report: fourth national climate assessment 1 470. https://doi.org/10.7930/J0J964J6

Zaller JR (1992). The nature and origins of mass opinion. Cambridge University Press

\section{Acknowledgements}

We are grateful for helpful discussions with and comments from Josh Horton.

\section{Competing interests}

The authors declare no competing interests.

\section{Additional information}

Supplementary information is available for this paper at https://doi.org/10.1057/s41599 020-00694-6.

Correspondence and requests for materials should be addressed to Z.D.

Reprints and permission information is available at http://www.nature.com/reprints

Publisher's note Springer Nature remains neutral with regard to jurisdictional claims in published maps and institutional affiliations.

Open Access This article is licensed under a Creative Commons Attribution 4.0 International License, which permits use, sharing, adaptation, distribution and reproduction in any medium or format, as long as you give appropriate credit to the original author(s) and the source, provide a link to the Creative Commons license, and indicate if changes were made. The images or other third party material in this article are included in the article's Creative Commons license, unless indicated otherwise in a credit line to the material. If material is not included in the article's Creative Commons license and your intended use is not permitted by statutory regulation or exceeds the permitted use, you will need to obtain permission directly from the copyright holder. To view a copy of this license, visit http://creativecommons.org/ licenses/by/4.0/

(C) The Author(s) 2021 